\title{
Esophageal Ulcers Associated with Ulcerative Colitis: A Case Series and Literature Review
}

\author{
Kentaro Tominaga ${ }^{1}$, Atsunori Tsuchiya ${ }^{1}$, Hiroki Sato ${ }^{1}$, Takeshi Mizusawa ${ }^{1}$, Shinichi Morita ${ }^{2}$, \\ Yui Ishii ${ }^{1}$, Nobutaka Takeda ${ }^{1}$, Kazuki Natsui ${ }^{1}$, Yuzo Kawata ${ }^{1}$, Naruhiro Kimura ${ }^{1}$, \\ Yoshihisa Arao ${ }^{1}$, Kazuya Takahashi ${ }^{1}$, Kazunao Hayashi ${ }^{1}$, Junji Yokoyama ${ }^{1}$ and Shuji Terai ${ }^{1}$
}

\begin{abstract}
:
Ulcerative colitis, a chronic and recurrent inflammatory disease, is localized to the colonic mucosa but can affect other organs and lead to various complications. Gastroduodenitis associated with ulcerative colitis has been reported. However, little is known about esophageal ulcers. We herein report two rare cases of esophageal ulcers associated with ulcerative colitis. Furthermore, the clinical and histological characteristics of 18 previously reported cases are summarized. This case series and literature review will encourage the accurate diagnosis and treatment of esophageal ulcers associated with ulcerative colitis.
\end{abstract}

Key words: ulcerative colitis, esophageal ulcer, manifestation

(Intern Med 59: 1983-1989, 2020)

(DOI: 10.2169/internalmedicine.4437-20)

\section{Introduction}

Ulcerative colitis (UC) is primarily a disease of the colon; however, extracolonic manifestations have been described. Extracolonic manifestations particularly involve the blood, joints, skin, biliary tracts, liver, kidneys, lungs, and upper digestive tract (1). Gastroduodenitis associated with UC (GDUC) has been noted in $7.6 \%$ of severe cases of $\mathrm{UC}(2,3)$, making it a common extracolonic manifestation of UC. In contrast, there have been relatively few reports of esophageal ulcer occurring as an extracolonic manifestation of UC (4).

We herein report two cases of UC complicated with esophageal ulcers. Furthermore, we review 18 previously reported cases of esophageal ulcers associated with UC. We summarize these cases to help characterize esophageal ulcer occurring as a complication of UC.

\section{Case Reports}

\section{Case 1}

A 16-year-old girl presented with chest pain and diarrhea with blood and mucus. No oral, genital ulcers or skin lesions were evident. Esophagogastroduodenoscopy (EGD), showed an ulcer with aphthae in the esophagus (Fig. 1a) and diffuse aphthae in the stomach (Fig. 1b). A histological examination of the esophageal ulcers showed severe inflammatory cell infiltration (Fig. 1d), and the gastric erosions revealed focally enhanced gastritis. Colonoscopy revealed superficial ulcers and mucosal friability (Fig. 1c) from the rectum to the cecum. The terminal ileum was normal. In the histological examination, inflammatory cell infiltration with cryptitis was detected in the sigmoid colon and rectum (Fig. 1e); therefore, pancolitis-type UC was diagnosed comprehensively.

She was not taking any medicine, including non-steroidal anti-inflammatory drugs, and Helicobacter pylori infection was not confirmed. A serological examination of viral infec-

\footnotetext{
${ }^{1}$ Division of Gastroenterology and Hepatology, Graduate School of Medical and Dental Sciences, Niigata University, Japan and ${ }^{2}$ Department of Gastroenterology and Hepatology, Uonuma Kikan Hospital, Japan

Received: January 8, 2020; Accepted: March 27, 2020; Advance Publication by J-STAGE: May 23, 2020

Correspondence to Dr. Atsunori Tsuchiya, atsunori@med.niigata-u.ac.jp
} 


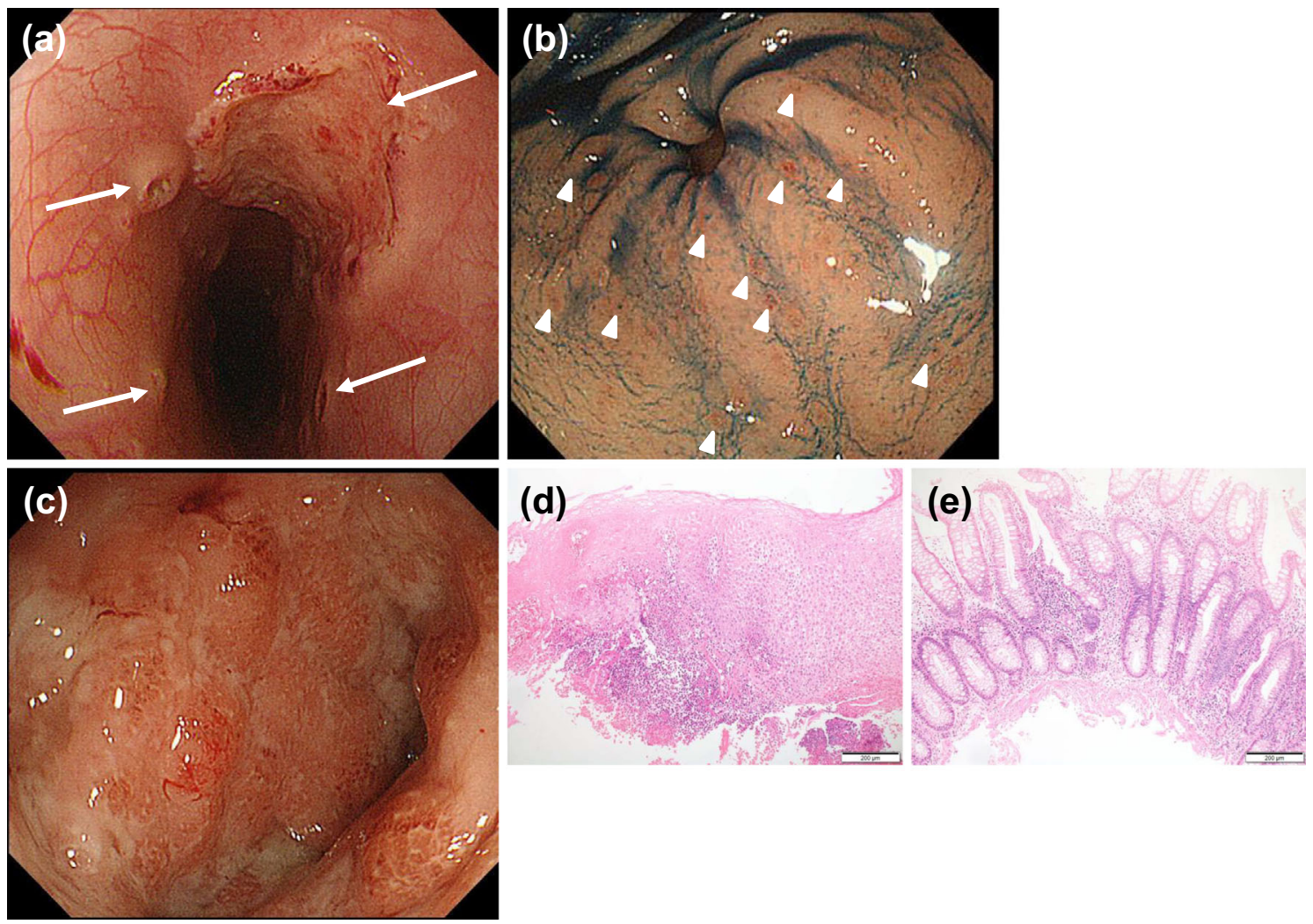

Figure 1. Esophagogastroduodenoscopy (EGD), colonoscopy, and their pathological findings. (a) EGD showing an ulcer with aphthae (arrows) in the mid intrathoracic esophagus. (b) EGD showing widespread aphthae (arrowheads) at the antrum. (c) Colonoscopy showing erythema and mucosal friability in the sigmoid colon. (d) Biopsy specimen from the esophageal ulcers showing severe inflammatory cell infiltration [Hematoxylin and Eosin $(H \& E)$ staining, $\times 100]$. (e) Biopsy specimen from the colon showing inflammatory cell infiltration with cryptitis in the rectum (H\&E staining, $\times 100)$.

tions (varicella zoster virus, herpes simplex virus, cytomegalovirus, and Epstein-Barr virus-DNA) was also negative. Therefore, esophageal ulcers and gastroduodenitis associated with UC (GDUC) were diagnosed.

Treatment with intravenous prednisolone (60 mg/day) was started, and her chest pain and diarrhea were resolved in a few days. EGD on the seventh day showed healing of the esophageal ulcers (Fig. 2a) and gastroduodenal aphthae (Fig. 2b). Colonoscopy showed improvement in the inflammation of the colon (Fig. 2c). She has experienced no relapse for two years.

\section{Case 2}

A 19-year-old woman was admitted to our hospital due to bloody stool and chest pain on swallowing. She was not taking any medication. Laboratory tests showed hypoalbuminemia, mild anemia and inflammatory responses: total protein (6.9 g/dL), albumin (2.8 g/dL), hemoglobin (10.1 g/dL), white blood cell $\left(4,140 / \mathrm{mm}^{3}\right)$, mild neutrophilic leukocytosis $(61.6 \%)$, thrombocytosis (platelets $28.9 \times 10^{4} / \mu \mathrm{L}$ ), and increased C-reactive protein levels $(6.88 \mathrm{mg} / \mathrm{dL})$. Stool cultures were normal. Serological assays for viruses, including herpes simplex, cytomegalovirus, varicella, and Epstein-Bar did not demonstrate primary infection. Because of her mouth and chest pain symptoms, EGD was performed, showing a longitudinal ulcer in the lower esophagus (Fig. 3a) and oral ulcers. Histologic findings showed infiltration of inflammatory cells in the epithelia (Fig. 3d). Colonoscopy showed superficial ulcers and mucosal friability (Fig. 3b) in the entire colon, from the rectum to the cecum. We also detected mucosal friability in the terminal ileum (Fig. 3c). The histologic findings here showed inflammatory cell infiltration with basal plasmacytosis in the transverse colon and inflammatory cell infiltration with cryptitis in the rectum (Fig. 3e). She was diagnosed with esophageal ulcer complicated with pancolitis-type UC comprehensively.

Induction therapy was started with intravenous prednisolone (50 mg/day). Thereafter, her bloody stool and pain on swallowing improved within one week. EGD after four weeks showed healing of the oral and esophageal ulcers (Fig. 4a). Colonoscopy after four weeks also showed complete remission (Fig. 4b). She has shown no relapse of esophageal lesions for one year.

\section{Discussion}

In 1961, Margoles et al. (5) first reported a case of pancolitis-type UC with esophagitis. There have been 18 re- 


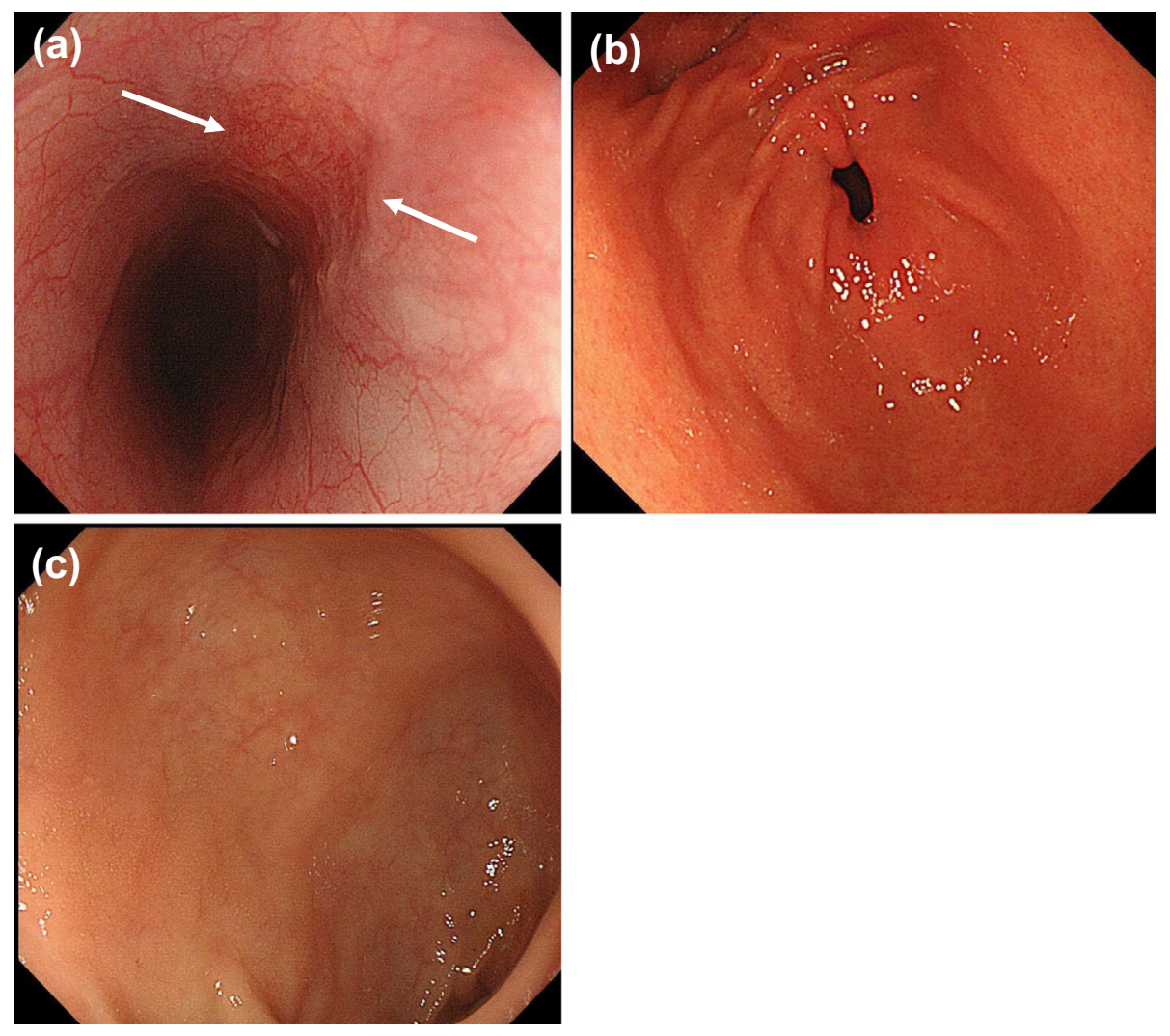

Figure 2. Esophagogastroduodenoscopy (EGD) and colonoscopic findings. (a) EGD showing marked healing of the esophageal ulcers (arrows). (b) EGD showing healing of the gastroduodenal aphthae. (c) Colonoscopy showing improvement of mucosal edema and vascular appearance of the sigmoid colon.

ported cases of esophageal ulcers associated with UC, and we encountered 2 new cases ourselves. The previously reported cases of esophageal ulcers associated with UC (case 3 to 20) and our new cases (case 1 and 2) are summarized in Tables 1-3.

We reviewed these 20 cases and summarized their findings in Table 1 (4-16). The average age of the patients was 28.2 years (range 14-53), and the ratio of men to women was 14:6. The duration of UC was from 0 to 21 years. There were 13 cases of pancolitis, 3 cases of left-side colitis, 1 case of right-side colitis, and 3 cases with unknown details. Seven of the 18 cases had esophageal ulcers at the onset of UC, and 2 cases had ulcers at the time of UC relapse. Two cases were in UC remission, and 7 cases occurred after total colectomy. According to the review results, esophageal ulcer complications were relatively common in the young compared with the elderly. Furthermore, esophageal ulcer was often complicated in patients at the onset of colitis, or at the time of relapse. It has been reported more aggressive UC entities, such as active pancolitis, may be related to the development of gastroduodenitis associated with UC (GDUC) (2). Furthermore, cases of esophageal ulcers after surgery indicate UC may not be resolved in other organs, even after total colectomy (3).

Of the 20 cases, there were 4 cases of multiple esophageal ulcers. There were two cases of entire esophageal ulcer, five of mid-esophageal ulcer, two of lower to middle esophageal ulcer, and five with unknown details. Endoscopic findings of esophageal ulcer complicated with UC were different from GDUC. While GDUC was defined as friable and granular mucosa $(2,3)$, esophageal ulcer complicated with UC is a punched-out ulcer and mostly occurs in the middle to the lower esophagus.

Regarding the pathology, in all cases described, only nonspecific inflammatory cell infiltration was observed, with no UC-specific findings reported. Although the relationship between the esophageal ulcers and UC was not confirmed histologically, no other causes of esophageal ulcers were identified, and treatment for UC was also effective for the esophageal lesions. Regarding treatment, 10 cases were treated with prednisolone, and 2 cases received 5-aminosalicylate. GDUC is treated with medications similar to those used for $\mathrm{UC}(3,17)$ and is more responsive to treatment, showing less frequent relapse than colitis. Our two newly reported cases followed a similar course.

Of the 18 reported cases, $45 \%$ had other complications, namely oral lesions in 7 cases, skin lesions in 6 cases, arthritis in 3 cases, and pancreatitis in 1 case. The case reported by Knudsen and Sparberg (6) had a generalized maculopapular eruption as a complication associated with pancolitis-type UC. The patient reported by Rosendorff (7) had buccal ulceration and arthritis. Most cases of UC with 


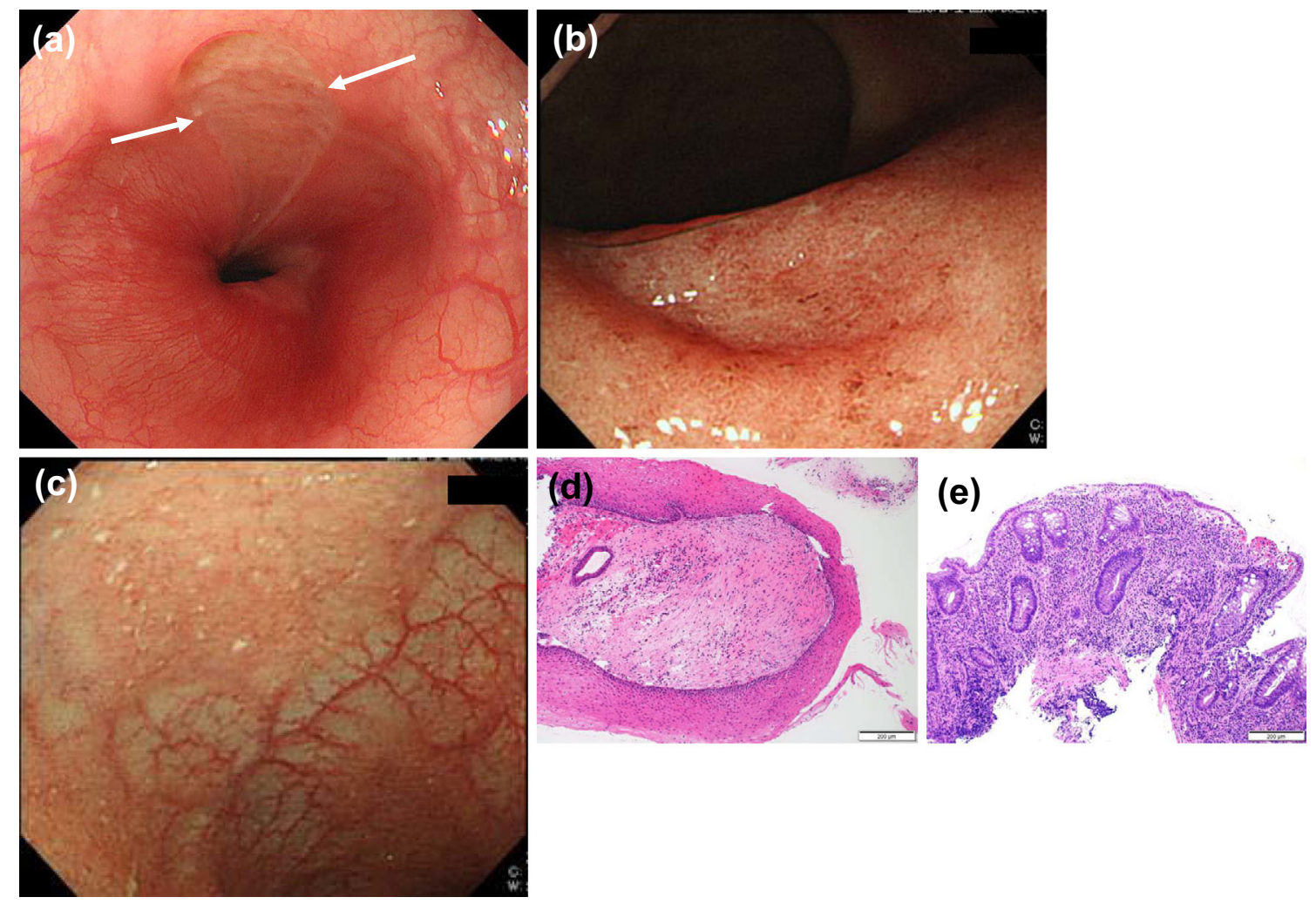

Figure 3. Esophagogastroduodenoscopy (EGD), colonoscopy, and their pathological findings. (a) EGD showing a longitudinal ulcer in the lower esophagus. (b) Colonoscopy showing mucosal friability in the rectum. (c) Colonoscopy showing mucosal friability in the terminal ileum. (d) Biopsy specimen from the esophageal ulcers showing infiltration of inflammatory cells in the epithelia [Hematoxylin and Eosin $(\mathrm{H} \& \mathrm{E})$ staining, $\times 100]$. (e) Biopsy specimen from the rectum showing inflammatory cell infiltration with basal plasmacytosis and cryptitis $(\mathrm{H} \& \mathrm{E}$ staining, $\times 100)$.
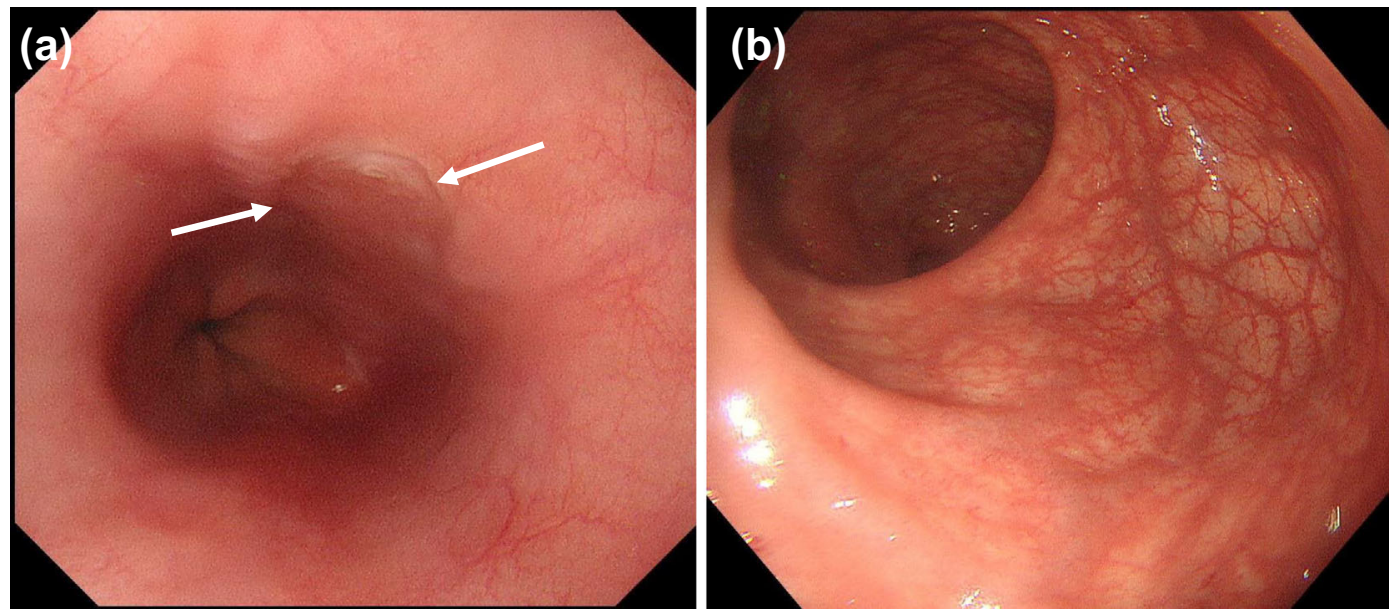

Figure 4. Esophagogastroduodenoscopy (EGD) and colonoscopic findings. (a) EGD showing healing of the esophageal ulcers. (b) Colonoscopy showing remission of the rectum.

esophageal ulcer have had other extracolonic manifestations. Various extracolonic manifestations of UC, such as ocular lesions, skin disease, and peripheral arthritis, have been reported (1); however, their causes are currently unknown.

In conclusion, esophageal ulcer associated with UC is extremely rare. In most cases, it may occur as an extracolonic manifestation. The pathological findings of esophageal le- sion are non-specific for UC. Therefore, a comprehensive diagnosis excluding disorders related to infection and medication is required. UC-associated esophageal ulcers are typically associated with the disease onset or relapse of UC. particularly in younger patients, and the treatment response is fortunately considered good. However, the etiology of esophageal ulcer associated with UC remains unknown. 
Table 1. Clinical Data of UC Patients with Esophageal Ulcer.

\begin{tabular}{lccccccc}
\hline $\begin{array}{c}\text { Case } \\
\text { (No) }\end{array}$ & $\begin{array}{c}\text { Ref } \\
\text { No. }\end{array}$ & $\begin{array}{c}\text { Age } \\
\text { (yrs) }\end{array}$ & Sex & $\begin{array}{c}\text { Duration } \\
\text { of UC }\end{array}$ & UC phase & Type & UC treatment \\
\hline 1 & Ours & 16 & F & 0 & Active & Total & PSL \\
2 & Ours & 19 & F & 0 & Active & Total & PSL \\
3 & 6 & 15 & M & 2 years & Remission & Left side & PSL, SASP \\
4 & 7 & 23 & M & 5 years & Remission & Total & PSL, SASP \\
5 & 8 & 21 & F & 0 & Active & N/A & PLS, SASP \\
6 & & 19 & M & 0 & Active & N/A & N/A \\
7 & 10 & 47 & F & 0 & Active & Right side & 5ASA \\
8 & 4 & 18 & F & 7 years & Relapse Active & Total & SASP \\
9 & 11 & 18 & M & 1 year & Active & Left side & PSL, 5ASA \\
10 & 12 & 19 & M & 0 & Active & Total & none \\
11 & 13 & 51 & M & 0 & Active & Total & PSL, 5ASA \\
12 & 14 & 33 & M & 0 & Active & Total & PSL, LCAP \\
13 & 15 & 52 & F & 3 years & Relapse Active & Left side & SASP \\
14 & 5 & 28 & M & 17 years & Post operation & Total & Total colectomy and ileostomy \\
15 & 8 & 26 & M & 2 months & Post operation & Total & Sbtotal colectomy and ileostomy \\
16 & & 14 & F & 5 years & Post operation & Total & Sbtotal colectomy and ileostomy \\
17 & & 24 & M & 9 years & Post operation & Total & Total proctocolectomy \\
18 & 9 & 21 & M & 3 years & Post operation & Total & Total colectomy and ileostomy \\
19 & 16 & 47 & M & 21 years & Post operation & N/A & Sbtotal colectomy and ileostomy \\
20 & & 53 & M & N/A & Post operation & Total & Sbtotal colectomy \\
\hline
\end{tabular}

UC: ulcerative colitis, N/A: data not available, PSL: prednisolone, SASP: salazosulfapyridine, 5-ASA: 5-aminosalicylic acid, F: female, M: male

Table 2. Clinical Data of UC Patients with Esophageal Ulcer.

\begin{tabular}{|c|c|c|c|}
\hline $\begin{array}{l}\text { Case } \\
(\mathrm{No})\end{array}$ & Esophagial ulcer (EU) & Location & EU symptoms \\
\hline 1 & An esophageal ulcer with aphthae & Middle esophagus & Chest pain \\
\hline 2 & Longitudinal oesophageal ulcer & Lower esophagus & Chest pain on swallowing \\
\hline 3 & Severe esophagitis & Lower esophagus & Parasternal chest pain and dysphagia \\
\hline 4 & $\begin{array}{c}\text { Shallow ulceration with gross diffuse irregularity } \\
\text { and polyp formation }\end{array}$ & Middle and lower esophagus & Dysphagia \\
\hline 5 & Active ulcerative esophagitis & N/A & N/A \\
\hline 6 & Marked ulcerative esophagitis & N/A & Substenal pain and dysphagia \\
\hline 7 & Multiple irregular esophageal ulcers & Whole esophagus & Anterior chest pain and dysphagia \\
\hline 8 & Longitudinal oesophageal ulcer with haemorrhage & Middle and lower esophagus & Sore throat and pain on swallowing \\
\hline 9 & Punched-out esophageal ulcer & Middle esophagus & Sore throat and anterior chest pain \\
\hline 10 & Punched-out esophageal ulcer & Middle esophagus & General fatigue \\
\hline 11 & Punched-out esophageal ulcer & Middle and lower esophagus & Anterior chest pain on swallowing \\
\hline 12 & Punched-out esophageal ulcer & Middle and lower esophagus & Anterior chest pain and dysphagia \\
\hline 13 & Punched-out esophageal ulcer & Middle esophagus & Anterior chest pain on swallowing \\
\hline 14 & Web formation & Middle esophagus & No \\
\hline 15 & $\begin{array}{c}\text { Esophagial ulcerations with perforaton into the } \\
\text { anterior mediastinum }\end{array}$ & N/A & Dysphagia, fever, hypotension \\
\hline 16 & Necrotizing fibrinopurulent ulceration & N/A & N/A \\
\hline 17 & Ulcerative and membranous esophagitis & N/A & Nausea, vomiting and hematemesis \\
\hline 18 & Multiple friable ulcerations & Whole esophagus & Severe odynophagia and dyspahgia \\
\hline 19 & Necrosis & Lower esophagus & Epigastric pain \\
\hline 20 & Necrosis & Middle and lower esophagus & Hematemesis \\
\hline
\end{tabular}

UC: ulcerative colitis, N/A: data not available

Since UC has various pathologies, the accumulation of more cases will be necessary to confirm these findings in the future. The two cases reported in the present study also had atypical aspects and require strict follow-up.

We believe that this case series and literature review will facilitate the accurate and prompt diagnosis of esophageal 
Table 3. Clinical Data of UC Patients with Esophageal Ulcer.

\begin{tabular}{|c|c|c|c|c|}
\hline $\begin{array}{l}\text { Case } \\
(\mathrm{No})\end{array}$ & EU biopsy & EU treatment & $\begin{array}{l}\text { Outcome of } \\
\text { EU }\end{array}$ & Other complication \\
\hline 1 & Severe inflammatory cell infiltration & PSL & Improvement & No \\
\hline 2 & $\begin{array}{l}\text { Infiltration of inflammatory cells in the } \\
\text { epithelia and no specific information }\end{array}$ & PSL & Improvement & Oral ulcer \\
\hline 3 & Superficial inflammation & PSL & Improvement & Maculopapular eruption \\
\hline 4 & $\begin{array}{c}\text { Chronically inflamed and edematous, with } \\
\text { a dense infltrate of plasma cells, } \\
\text { lymphocytes, and histocytes }\end{array}$ & PSL & Improvement & Buccal ulceration and arthritis \\
\hline 5 & N/A & - & - & No \\
\hline 6 & N/A & - & - & $\begin{array}{l}\text { Necrotic ulceration in the inguinal, } \\
\text { genital regions and lower extremities }\end{array}$ \\
\hline 7 & $\begin{array}{l}\text { Nonspecific findings other than } \\
\text { inflammatorycell infiltration }\end{array}$ & 5ASA, H2blocker & Improvement & No \\
\hline 8 & $\begin{array}{l}\text { Nonspecific findings other than } \\
\text { inflammatorycell infiltration }\end{array}$ & PSL & Improvement & No \\
\hline 9 & $\begin{array}{l}\text { Nonspecific findings (Appearance of } \\
\text { regenerative epithelium) }\end{array}$ & PSL & Improvement & Oral ulcer and pancreatitis \\
\hline 10 & Nonspecific findings & PSL, SASP & Improvement & No \\
\hline 11 & $\begin{array}{l}\text { Thickening of epithelial stratum spinosum } \\
\text { without nuclear inclusion body }\end{array}$ & PPI & Improvement & No \\
\hline 12 & $\begin{array}{l}\text { Nonspecific findings (Appearance of } \\
\text { regenerative epithelium) }\end{array}$ & PPI & Improvement & No \\
\hline 13 & $\begin{array}{l}\text { Nonspecific findings other than } \\
\text { inflammatorycell infiltration }\end{array}$ & PSL & Improvement & No \\
\hline 14 & N/A & PSL & N/A & Mouth and pyoderma gangrenosum \\
\hline 15 & N/A & - & - & $\begin{array}{l}\text { Oral ulcerations, pustular dermatitis, } \\
\text { arthritis }\end{array}$ \\
\hline 16 & N/A & - & - & N/A \\
\hline 17 & N/A & - & - & $\begin{array}{c}\text { Ulcar of his buttock, paronychia of } \\
\text { hands }\end{array}$ \\
\hline 18 & $\begin{array}{l}\text { Dense infiltrate of polymorphonuclear cells, } \\
\text { a few eosinophils and mononuclear cells }\end{array}$ & PSL, tetracycline & N/A & $\begin{array}{l}\text { Arthritis, episcleritis, oral ulcerations } \\
\text { and erythema nodosum }\end{array}$ \\
\hline 19 & Inflammatory cell and infiltration & PPI & Stricture & No \\
\hline 20 & Not examination & PPI & Stricture & No \\
\hline
\end{tabular}

ulcer associated with UC.

Informed consent was obtained from the patients for the publication of their information and imaging.

The authors state that they have no Conflict of Interest (COI).

\section{Financial Support}

This work was supported by Takeda Japan Medical Office Funded Research Grant 2018.

\section{References}

1. Greenstein AJ, Janowits HD, Sachar DB. The extra-intestinal complications of Crohn's disease and ulcerative colitis: a study of 700 patients. Medicine (Baltimore) 55: 401-412, 1976.

2. Hori K, Ikeuchi $\mathrm{H}$, Nakano $\mathrm{H}$, et al. Gastroduodenitis associated with ulcerative colitis. J Gastroenterol 43: 193-201, 2008.

3. Hisabe T, Matsui T, Miyaoka M, et al. Diagnosis and clinical course of ulcerative gastroduodenal lesion associated with ulcerative colitis: possible relationship with pouchitis. Dig Endosc 22:
268-274, 2010.

4. Askawa A, Kojima Y, Fujii E, et al. Case of ulcerative colitis associated with oesophageal ulcer. J Int Med Res 28: 191-196, 2000.

5. Margoles JS, Wenger J. Stomal ulceration associated with pyoderma gangrenosum and chronic ulcerative colitis. Report of two cases. Gastroenterology 41: 594-598, 1961.

6. Knudsen KB, Sparberg M. Ulcerative oesophagitis and ulcerative colitis. JAMA 201: 140, 1967.

7. Rosendorff C, Grieve NW. Ulcerative oesophagitis in association with ulcerative colitis. Gut 8: 344-347, 1967.

8. Christopher NL, Watson DW, Farber ER. Relationship of chronic ulcerative oesophagitis to ulcerative colitis. Ann Intern Med 70: 971-976, 1969.

9. Haward MZ, Gilbert R, Simmy B. Apthous ulcers of the esophagus in a patient with ulcerative colitis. Gastrointest Endosc 30: 298-299, 1984.

10. Konishi H, Mitsuhashi Y, Fujino N, et al. A case of right-sided ulcerative colitis associated with unusual esophageal ulcer. Chiba Medical Association Magazine 50: 143-144, 1998 (in Japanese).

11. Ikeda Y, Yonezawa K, Suzuki K, et al. A case of ulcerative colitis associated with a hemorrhagic esophageal ulcer. J Kushiro City Gen Hosp 12: 129-133, 2000 (in Japanese).

12. Higashi Y, Nakamura T, Sunayama K, et al. A case of ulcerative 
colitis combined with esophageal ulcer. Nihon Rinsho Geka Gakkai Zasshi (J Japan Surg Assoc) 65: 143-146, 2004 (in Japanese, Abstract in English).

13. Sato N, Kurakata H, Hirano N, et al. A case of ulcerative colitis combined with esophageal ulcer. Progress of Digestive Endoscopy 65: 90-91, 2004 (in Japanese).

14. Ose $\mathrm{T}$, Ishihara $\mathrm{S}$, Oshima $\mathrm{N}$, et al. A case of ulcerative colitis complicated with giant esophageal ulcer. Clinics in Gastroenterology 11: 93-96, 2005.

15. Izawa N, Sugaya $T$, Kanamori A, et al. A case of ulcerative colitis with esophageal ulcer. Progress of Digestive Endoscopy 87: 94-95, 2015 (in Japanese, Abstract in English).
16. Kuroki H, Sugita A, Koganei K, et al. Two cases of esophageal ulcer after surgical treatment for ulcerative colitis. Clin J Gastroenterol. Forthcoming.

17. Chiba M, Ono I, Wakamatsu H, Wada I, Suzuki K. Diffuse gastroduodenitis associated with ulcerative colitis: treatment by infliximab. Dig Endosc 25: 622-625, 2013.

The Internal Medicine is an Open Access journal distributed under the Creative Commons Attribution-NonCommercial-NoDerivatives 4.0 International License. To view the details of this license, please visit (https://creativecommons.org/licenses/ by-nc-nd/4.0/).

(C) 2020 The Japanese Society of Internal Medicine Intern Med 59: 1983-1989, 2020 